\title{
Excitation of Molecular Vibrational Modes with Inelastic Scanning Tunneling Microscopy Processes: Examination through Action Spectra of cis-2-Butene on Pd(110)
}

\author{
Yasuyuki Sainoo, ${ }^{1, *}$ Yousoo Kim, ${ }^{1, \dagger, \ddagger}$ Toshiro Okawa, ${ }^{2}$ Tadahiro Komeda, ${ }^{3,6}$ \\ Hidemi Shigekawa, ${ }^{4,6}$ and Maki Kawai ${ }^{1,5,6, \uparrow, 8}$ \\ ${ }^{1}$ RIKEN, 2-1 Hirosawa, Wako, Saitama 351-0198, Japan \\ ${ }^{2}$ Department of Physics, Gakushuin University, Mejiro, Toshima-ku, Tokyo 171-8588, Japan \\ ${ }^{3}$ Institute of Multidisciplinary Research for Advanced Materials, Tohoku University, 2-1-1 Katahira, Aoba, Sendai 980-8577, Japan \\ ${ }^{4}$ Institute of Applied physics, 21th Century COE, NANO project, University of Tsukuba, 305-8573, Japan \\ ${ }^{5}$ Department of Advanced Materials Science, The University of Tokyo, Kashiwa, Chiba 277-8651, Japan \\ ${ }^{6}$ CREST, Japan Science and Technology Corporation (JST), Toshima-ku, Tokyo 171-0031, Japan
}

(Received 8 March 2005; published 5 December 2005)

\begin{abstract}
Inelastically tunneled electrons from a scanning tunneling microscope (STM) were used to induce vibrationally mediated motion of a single cis-2-butene molecule among four equivalent orientations on $\operatorname{Pd}(110)$ at $4.8 \mathrm{~K}$. The action spectrum obtained from the motion clearly detects more vibrational modes than inelastic electron tunneling spectroscopy with a STM. We demonstrate the usefulness of the action spectroscopy as a novel single molecule vibrational spectroscopic method. We also discuss its selection rules in terms of resonance tunneling.
\end{abstract}

DOI: 10.1103/PhysRevLett.95.246102

PACS numbers: 68.35.Fx, 63.22.+m, 68.37.Ef, 82.65.+r

The excitation of molecular vibration by means of the inelastically tunneled electrons from the tip of a scanning tunneling microscope (STM) can lead to various dynamical processes at surfaces [1,2]. In addition, inelastic electron tunneling spectroscopy with the STM (STM-IETS) [3] is now applicable to the vibrational spectroscopy of the individual molecules. The vibrational spectrum of a single molecule provides useful information not only for the chemical identification of the molecule [4-6], but also for investigating how molecular vibration can couple with the relevant dynamical processes $[7,8]$.

However, many experimental results [1,3-8] have shown that not all the vibrational modes are observed in STM-IET spectra, which indicates that the establishment of proper selection rules is necessary. In order to establish the selection rules, it is of crucial importance to clarify experimental observation of the molecular vibration induced by inelastically tunneled electrons. STM-IETS detects the vibrational modes of a single molecule by measuring the total conductance change resulting from the inelastic electron tunneling. However, the total conductance change is not always detectable, since the elastic component can be adversely reduced giving rise to a decrease in IET signals for some vibrational modes [9]. The response of vibrationally mediated molecular motion to applied bias voltage, namely, an "action spectrum," can reveal vibrational modes that are not visible in STM-IETS, because the molecular motion is induced via only inelastic tunneling. Thus, the action spectrum would be a candidate for detecting which vibrational mode is actually excited and associated with molecular motions. Here, we show the usefulness of action spectroscopy as an alternative vibrational spectroscopic method for the STM-IETS through the study of vibrationally mediated reversible motion of a cis-2-butene molecule among four equivalent adsorption orientations on $\mathrm{Pd}(110)$ at $4.8 \mathrm{~K}$. The STM-IET spectrum shows only two peaks corresponding to the metal-carbon stretch mode, $\nu(M-\mathrm{C})$, and the $\mathrm{CH}$ stretch mode in the $\mathrm{CH}_{3}$ group, $\nu\left(\mathrm{CH}_{3}\right)$. However, the action spectrum reveals that not only $\nu(M-\mathrm{C})$ and $\nu\left(\mathrm{CH}_{3}\right)$ but also the $\mathrm{C}-\mathrm{C}$ stretch mode, $\nu(\mathrm{C}-\mathrm{C})$, and the bending mode in $\mathrm{CH}_{3}, \delta\left(\mathrm{CH}_{3}\right)$, are actually excited via inelastic tunneling leading to the motions.

All experiments were performed using a commercially available low-temperature STM (LT-STM, Omicron $\mathrm{GmbH})$ equipped in an ultrahigh-vacuum chamber $(<3 \times$ $\left.10^{-9} \mathrm{~Pa}\right)[8,10]$. The $\mathrm{Pd}(110)$ surface was cleaned by cycles of $\mathrm{Ar}$ ion sputtering and annealing cycles, and exposed to cis-2-butene molecules below $50 \mathrm{~K}$. All images and spectroscopic data were acquired at $4.8 \mathrm{~K}$; experimental details for spectroscopy have been reported elsewhere $[8,11,12]$.

A cis-2-butene molecule adsorbed on $\operatorname{Pd}(110)$ appears as a gourd shape in the STM image with a large bright region (head) and a small less bright region in Fig. 1(a). There are four equivalent orientations, labeled $C_{\mathrm{UR}}, C_{\mathrm{UL}}, C_{\mathrm{DR}}$, and $C_{\mathrm{DL}}$. An isolated cis-2-butene is $\pi$-bonded to off-centered position of the $\mathrm{Pd}$ atom, where the molecule is slightly shifted towards the hollow site [11-13]. The adsorption geometry is proposed as shown in Fig. 1(b).

The procedure for inducing molecular motion is described previously [12]. In brief, after imaging the target molecule at initial orientation, the STM tip is positioned over the center of the head part of the molecule. Then, the bias voltage is increased to a certain value and the tunneling current is recorded as a function of time. A rescanning of the same area shows that the molecule has changed its orientations one to the other. The repeated sequences show 
(a)

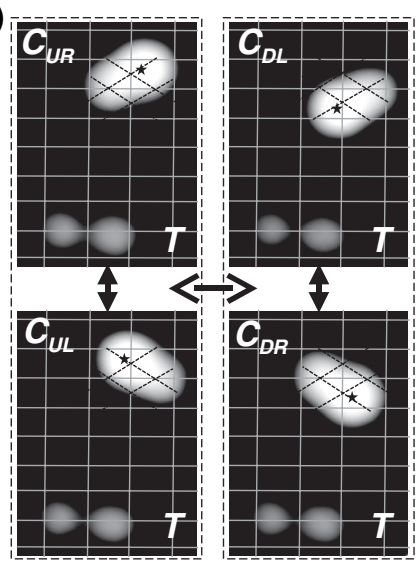

(b)

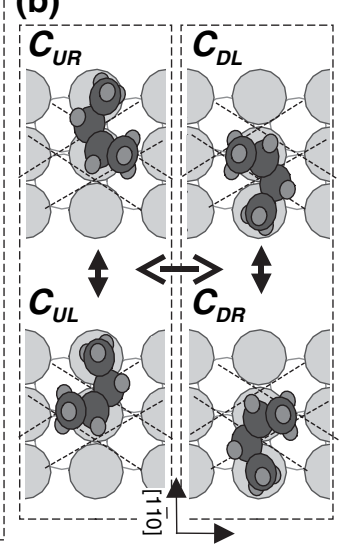

(c)

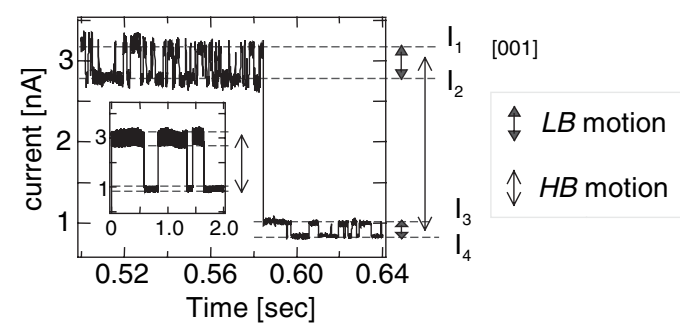

FIG. 1. (a) STM images of four equivalent orientations of a cis-2-butene molecule labeled $C_{\mathrm{UR}}, C_{\mathrm{DR}}, C_{\mathrm{UL}}$, and $C_{\mathrm{DL}}$, which are obtained by moving the molecule by injecting tunneling electrons. The suffixes UR, UL, DR, and DL correspond to the relative location of the head part, i.e., up-right, up-left, downright, and down-left, with respect to the center of the molecule in the STM images, respectively. A trans-2-butene molecule (labeled $T$ ) was coadsorbed as a marker. The superimposed grid indicates the position of Pd atoms, which is determined from the STM image obtained with a molecular tip. The additional four dotted lines forming a rhombus correspond to the molecular long axes at each orientation. An asterisk represents the center of the large bright region of the molecule in each orientation, where the tunneling electrons were injected. (b) Proposed structure of cis2-butene on $\operatorname{Pd}(110)$ at four equivalent adsorption orientations. (c) Current changes measured at sample bias voltage of $170 \mathrm{mV}$ with the tip fixed over the center of the head of a cis-2-butene molecule. The inset shows the wide time range of the same data.

that the trace of tunneling current reflects residence time of the molecule at a certain orientation. Figure 1(c) shows a typical change in tunneling current while the sample bias voltage is kept constant at $170 \mathrm{mV}$.

The motions of cis-2-butene can be categorized into two types [12]; they are extracted from the difference in current changes and simplified by defining the pairs of $\left(C_{\mathrm{UR}}, C_{\mathrm{UL}}\right)$ and of $\left(C_{\mathrm{DR}}, C_{\mathrm{DL}}\right)$; the pairs are indicated by arrows in Figs. $1(\mathrm{a})$ and $1(\mathrm{~b}) . C_{\mathrm{UR}}$ and $C_{\mathrm{UL}}\left(C_{\mathrm{DR}}\right.$ and $\left.C_{\mathrm{DL}}\right)$ are mirror images with respect to a plane parallel to [110] , while $C_{\mathrm{UR}}$ and $C_{\mathrm{DR}}\left(C_{\mathrm{UL}}\right.$ and $\left.C_{\mathrm{DL}}\right)$ are also mirror images with respect to a plane parallel to [001]. The motion with small changes in current, such as either between $I_{1}$ and $I_{2}$ or between $I_{3}$ and $I_{4}$ in Fig. 1(c), corresponds to the change between two orientations with the mirror plane parallel to [110]. This motion is named "low barrier (LB)" motion. The other motion is named "high barrier (HB)" motion with larger current change between $\left(I_{1}, I_{2}\right)$ and $\left(I_{3}, I_{4}\right)$ in Fig. 1(c), corresponding to the orientation change between two pairs for LB motion. The potential barrier for $\mathrm{HB}$ motion is higher than that of LB motion, which is clearly seen in action spectra.

Figure 2 shows the action spectra of cis-2-butene, where the motion yield (number of molecular motions per injected electron) is indicated as a function of applied sample bias voltage at a chosen tunneling current. There are clear thresholds for both $\mathrm{LB}$ and $\mathrm{HB}$ motions of $\mathrm{C}_{4} \mathrm{H}_{8}$ in the upper spectra of Fig. 2(a). For LB motion, the motion yield markedly increased at $\sim 37 \mathrm{mV}$ followed by a slight increase at $\sim 115 \mathrm{mV}$. For HB motion, on the other hand, clear increases were observed at $\sim 115$ and $\sim 360 \mathrm{mV}$. A comparison between $\mathrm{C}_{4} \mathrm{H}_{8}$ and fully deuterated cis-2butene $\left(\mathrm{C}_{4} \mathrm{D}_{8}\right)$ helps to assign the active vibrational modes to the above-mentioned motion. In the action spectrum of $\mathrm{C}_{4} \mathrm{D}_{8}$, the increases of the motion yield for LB motion were observed at $\sim 31 \mathrm{mV}$ and at $\sim 95 \mathrm{mV}$. For HB motion, the increases of the motion yield appeared at $\sim 110 \mathrm{mV}$ and at $\sim 270 \mathrm{mV}$. The assignment for those modes is described below with combing to STM-IETS, which gives us the vibrational signature of an individual molecule.

Figure 3 shows the STM-IET spectra of $\mathrm{C}_{4} \mathrm{H}_{8}$ [Fig. 3(a)] and $\mathrm{C}_{4} \mathrm{D}_{8}$ [Fig. 3(b)]. The significant features appear at

(a)

(b)
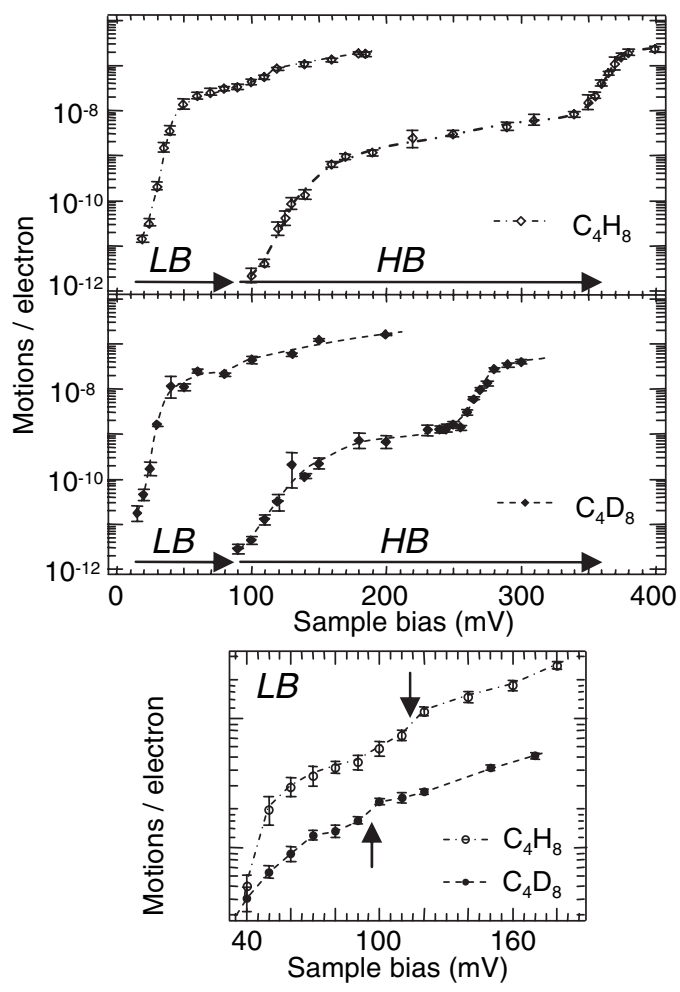

FIG. 2. (a) Action spectra for described motions both of $\mathrm{C}_{4} \mathrm{H}_{8}$ (upper) and of $\mathrm{C}_{4} \mathrm{D}_{8}$ (lower). Data were taken under fixed tunneling current of $3 \mathrm{nA}$ for $\mathrm{C}_{4} \mathrm{H}_{8}$ and of $2 \mathrm{nA}$ for $\mathrm{C}_{4} \mathrm{D}_{8}$. (b) Magnification of the action spectrum for low barrier motion at around the threshold energy. Slight increase in the yield was observed around $115 \mathrm{mV}$ for $\mathrm{C}_{4} \mathrm{H}_{8}$ and $95 \mathrm{mV}$ for $\mathrm{C}_{4} \mathrm{D}_{8}$, as indicated by arrows, respectively. 

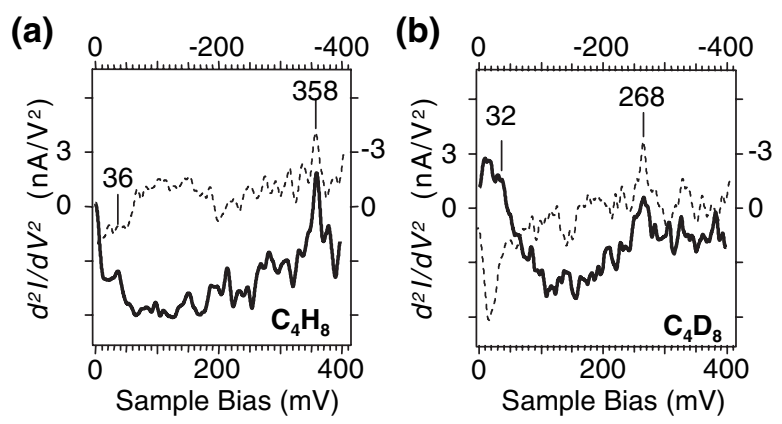

FIG. 3. STM-IETS spectra taken over (a) $\mathrm{C}_{4} \mathrm{H}_{8}$ and (b) $\mathrm{C}_{4} \mathrm{D}_{8}$. Solid lines and dashed lines represent vibrational spectra of the positive and negative bias range, respectively. The background spectrum taken over bare $\operatorname{Pd}(110)$ surface has been subtracted from each spectrum. All spectra were obtained at a tunneling gap set point of $0.25 \mathrm{nA}$ and $20 \mathrm{mV}$, and $V_{\text {rms }}=15 \mathrm{mV}$ ac modulation at $797 \mathrm{~Hz}$. Each spectrum is the average of 16 bias voltage ramps.

$\pm 358 \mathrm{mV}$ for $\mathrm{C}_{4} \mathrm{H}_{8}$ and at $\pm 268 \mathrm{mV}$ for $\mathrm{C}_{4} \mathrm{D}_{8}$. Identical signals were observed in the STM-IET spectra of trans-2butene on $\mathrm{Pd}(110)$, and they were assigned to the vibrational peaks for $\nu\left(\mathrm{CH}_{3}\right)$ and $\nu\left(\mathrm{CD}_{3}\right)$, respectively [8]. An additional feature with a very low intensity at about 36 (32) $\mathrm{mV}$ for $\mathrm{C}_{4} \mathrm{H}_{8}\left(\mathrm{C}_{4} \mathrm{D}_{8}\right)$ was observed repeatedly for different sets of molecule and tip. No vibrational feature other than the two peaks was observable in the STM-IET spectra.

The high-resolution electron energy loss spectroscopy (HREELS) spectrum of cis-2-butene on $\operatorname{Pd}(110)$ [13] presents four groups of vibrational energies observed in different regions: (1) metal-carbon stretching mode at 20$40 \mathrm{meV}$; (2) $\mathrm{CH}$ bending and $\mathrm{C}-\mathrm{C}$ stretching modes at approximately $100 \mathrm{meV}$; (3) $\mathrm{C}=\mathrm{C}$ stretching mode, $\nu(\mathrm{C}=\mathrm{C})$, at $160-180 \mathrm{meV}$; and (4) $\mathrm{CH}$ stretching mode at approximately $360 \mathrm{meV}$.

Comparing with the HREELS spectrum, it turns out that both the first threshold in the action spectrum for LB motion of $\mathrm{C}_{4} \mathrm{H}_{8}\left(\mathrm{C}_{4} \mathrm{D}_{8}\right)$ observed at 37 (31) $\mathrm{mV}$ and the small peak observed in STM-IET spectrum at 36 (32) $\mathrm{mV}$ correspond to the vibrational energy of $\nu(M-\mathrm{C})$. Thus, we conclude that the excitation of $\nu(M-\mathrm{C})$ directly couples with LB motion. For region (2), while the lower threshold for $\mathrm{HB}$ motion was found at $\sim 110 \mathrm{mV}$ both for $\mathrm{C}_{4} \mathrm{H}_{8}$ and for $\mathrm{C}_{4} \mathrm{D}_{8}$ [Fig. 2(a)], a clear isotopic shift was observed from $115\left(\mathrm{C}_{4} \mathrm{H}_{8}\right)$ to $95 \mathrm{mV}\left(\mathrm{C}_{4} \mathrm{D}_{8}\right)$ for $\mathrm{LB}$ motion in Fig. 2(b). The latter case can be explained with the isotope shift of $\delta\left(\mathrm{CH}_{3}\right)$ and $\delta\left(\mathrm{CD}_{3}\right)$. On the other hand, $\nu(\mathrm{C}-\mathrm{C})$ should be expected to show a weak isotope shift. Thus, these two modes overlap in energy for $\mathrm{C}_{4} \mathrm{H}_{8}$ but are separated for $\mathrm{C}_{4} \mathrm{D}_{8}$, and both modes contribute to inducing molecular motion. For region (4), it is obvious that $\nu\left(\mathrm{CH}_{3}\right)$ corresponds to both vibrational signals, observed at \pm 358 (268) $\mathrm{mV}$ in the STM-IETS spectrum and at $\sim 360$ $(\sim 270) \mathrm{mV}$ in the action spectrum of $\mathrm{HB}$ motion of $\mathrm{C}_{4} \mathrm{H}_{8}\left(\mathrm{C}_{4} \mathrm{D}_{8}\right)$. However, $\nu(\mathrm{C}=\mathrm{C})$ in region (3) was invisible in both the STM-IETS and the action spectrum. A similar behavior was observed in the hopping motion of $\mathrm{C}_{2} \mathrm{H}_{4}$ on $\mathrm{Pd}(110)$, where no response was observed in the $\mathrm{C}=\mathrm{C}$ stretching mode region [14].

From the above-mentioned analysis of the action spectra, it is clear that the vibrational modes $\nu(M-\mathrm{C}), \delta\left(\mathrm{CH}_{3}\right)$, $\nu(\mathrm{C}-\mathrm{C})$ and $\nu\left(\mathrm{CH}_{3}\right)$ are excited via the inelastic electron tunneling. Since the $\nu(M-\mathrm{C})$ mode is directly related to the motion of the molecule, the excitation of all the higher vibrational modes can induce the same motion in their relaxation processes. The activation barrier for the motion of the molecule discussed here is pretty small, and the result of motion rate measurement as a function of applied current indicates that the excitation of two quanta of $\nu(\mathrm{C}-M)$ is sufficient to overcome the barrier [15]. However, although the stretching mode and the bending mode of $s p^{3} \mathrm{CH}$ in $\mathrm{CH}_{3}$ groups were clearly observed in the action spectra, those for $s p^{2} \mathrm{CH}$ at the $\mathrm{C}=\mathrm{C}$ bond were not observed. Note that $\nu(=\mathrm{CH})$ and $\delta(=\mathrm{CH})$ for $s p^{2} \mathrm{CH}$ appear in regions of different energies from those of $s p^{3} \mathrm{CH}$ 's, typically at $380 \mathrm{meV}$ and at $80 \mathrm{meV}$, respectively, in HREELS spectrum $[13,16]$. In order to explain this issue, we discuss the mechanism of the excitation of molecular vibration modes in STM via the inelastic electron tunneling.

Within an STM junction, electrons from the tip first encounter the molecular orbital at the lowest unoccupied molecular orbital (LUMO) level forming a temporary negative-ion state, inducing slight distortion of the molecule [17]. Since the lifetime of the temporary negative-ion state should be extremely short, the distorted molecule in the electronic ground state remains. The actual LUMO state of the adsorbate is the hybridized state, consisting of the highest occupied molecular orbital (HOMO) state, the LUMO state of the isolated molecule, and the $d$ orbitals of Pd. Even so, the distortion in the molecule should reflect the shapes of HOMO and LUMO of the isolated molecule unless the molecule is significantly distorted upon adsorption.

The shapes of HOMO and LUMO of a cis-2-butene molecule are depicted in Fig. 4. Both HOMO and LUMO have significant parts at the $\pi$ bonding and antibonding orbitals, and at the $s p^{3}$ hydrogen atoms, but not at $s p^{2}$ hydrogen atoms. Indeed, the action spectra revealed that the motion of cis-2-butene was actually enhanced for $\delta\left(\mathrm{CH}_{3}\right)$ but was inactive for $\delta(\mathrm{CH})$, corresponding to the fact that the molecular orbitals of cis-2-butene have a distribution at $s p^{3} \mathrm{CH}$ in methyl groups but not at $s p^{2}$ $\mathrm{CH}$ at the $\mathrm{C}=\mathrm{C}$ bond. Because of the distribution of the electronic state, $\delta(\mathrm{CH})$ was not directly excited via inelastic electron tunneling process even though the energy of the electron was sufficient for the excitation.

Although the $\mathrm{C}=\mathrm{C}$ bond is strongly associated with the adsorption states through both HOMO and LUMO, there were no signs of the excitation of $\nu(\mathrm{C}=\mathrm{C})$ in the action spectrum. Response to the molecular motion is only available if the vibrational mode can couple with the mode of reaction coordinate for the motion via anharmonic coupling. Since cis-2-butene is $\pi$ bonded to the Pd atom, 


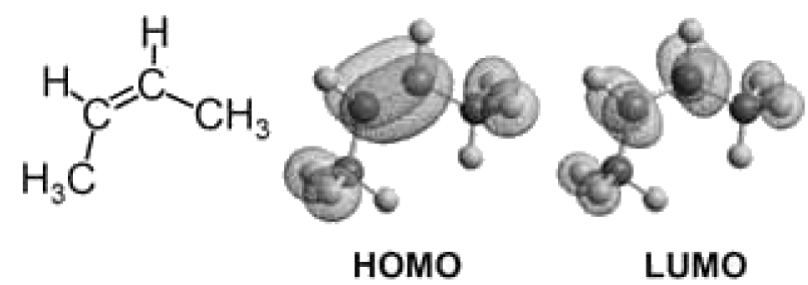

FIG. 4. Molecular structure (left), and the shapes of HOMO (center) and LUMO (right) of a cis-2-butene molecule. The molecular orbitals (MO) were calculated using a semiempirical quantum-mechanical calculation package MOPAC.

$\nu(\mathrm{C}=\mathrm{C})$ is strongly coupled with the electronic states near the Fermi level. Thus the vibrational damping through electron-phonon pair excitation could be quite efficient [18]. Despite that the energy is sufficient to overcome the barrier for the molecular motion, $\nu(\mathrm{C}=\mathrm{C})$ is invisible in the action spectra of cis-2-butene due to the insufficient coupling between vibrational modes similar to the case of $\mathrm{CO}$ on $\mathrm{Cu}(110)$ [10].

We have discussed the plausible mechanism of vibrational excitation via inelastic electron tunneling in the STM junction by the use of STM-IETS and action spectroscopy for the vibrationally mediated molecular motion of cis-2-butene. Taking the action spectrum into account, we have shown here that such an excitation is basically through resonant tunneling $[2,17,19,20]$. In other words, the coupling of the vibrational excitation to molecular motion reflects the feature of the LUMO of the adsorption state. For cis-2-butene on $\operatorname{Pd}(110), \nu(M-\mathrm{C}), \delta\left(\mathrm{CH}_{3}\right)$, $\nu(\mathrm{C}-\mathrm{C})$, and $\nu\left(\mathrm{CH}_{3}\right)$ are found to be excited, while only $\nu(M-\mathrm{C})$ and $\nu\left(\mathrm{CH}_{3}\right)$ are observed in STM-IETS. In order to understand the reason why the other modes are not observed in STM-IETS, a precise theoretical calculation would be necessary; even though it has been claimed that the elastic component contribute to diminish the inelastic component when the density of states is very high at the Fermi level $[9,19]$.

The determination of which vibrational mode is responsible for and how they are associated with the various surface dynamics is essential for achieving mode-selective control of chemical reactions. A combination of a dynamic spectroscopic technique such as an action spectroscopy and a static technique as an STM-IETS would give an important information to establish the actual dynamics related to the electron-vibration coupling in a single molecule on the surface.

The authors thank Dr. Hirokazu Fukidome and Dr. Satoshi Katano for valuable discussion on the assignment of vibrational modes. The present work was supported, in part, by the Grant-in-Aid for Scientific Research on Priority Areas "Electron transport through a linked molecule in nano-scale" (Grant No. 17069006) from the Ministry of Education, Culture, Sports, Science and Technology and International Joint Research Grant
"Molecular wire" project (03BR1) from the New Energy Development Organization (NEDO) of Japan.

*Present address: Institute of Multidisciplinary Research for Advanced Materials, Tohoku University, 2-1-1 Katahira, Aoba, Sendai 980-8577, Japan.

${ }^{\dagger}$ The authors to whom correspondence should be addressed. Email address: ykim@ riken.jp

${ }^{\S}$ Email address: maki@ riken.jp

[1] W. Ho, J. Chem. Phys. 117, 11033 (2002), and references therein; T. Komeda, Prog. Surf. Sci. 78, 41 (2005), and references therein.

[2] H. Ueba, Surf. Rev. Lett. 10, 771 (2003), and references therein; N. Lorente, R. Rurali, and H. Tang, J. Phys. Condens. Matter 17, S1049 (2005), and references therein.

[3] B. C. Stipe, M. A. Rezaei, and W. Ho, Science 280, 1732 (1998); Phys. Rev. Lett. 82, 1724 (1999).

[4] J. Gaudioso, H. J. Lee, and W. Ho, J. Am. Chem. Soc. 121, 8479 (1999); L. J. Lauhon and W. Ho, Phys. Rev. B 60, R8525 (1999); J. Phys. Chem. A 104, 2463 (2000).

[5] H. J. Lee and W. Ho, Science 286, 1719 (1999); J.R. Hahn, H. J. Lee, and W. Ho, Phys. Rev. Lett. 85, 1914 (2000); J. R. Hahn and W. Ho, Phys. Rev. Lett. 87, 166102 (2001).

[6] J. I. Pascual et al., Phys. Rev. Lett. 86, 1050 (2001).

[7] B. C. Stipe, M. A. Rezaei, and W. Ho, Phys. Rev. Lett. 81, 1263 (1998).

[8] Y. Kim, T. Komeda, and M. Kawai, Phys. Rev. Lett. 89, 126104 (2002).

[9] B. N. J. Persson and J.E. Demuth, Solid State Commun. 57, 769 (1986); N. Mingo and K. Makoshi, Phys. Rev. Lett. 84, 3694 (2000); N. Lorente and M. Persson, Phys. Rev. Lett. 85, 2997 (2000); F. E. Olsson, M. Persson, N. Lorente, L. J. Lauhon, and W. Ho, J. Phys. Chem. B 106, 8161 (2002).

[10] T. Komeda et al., Science 295, 2055 (2002).

[11] Y. Sainoo, Y. Kim, T. Komeda, and M. Kawai, J. Chem. Phys. 120, 7249 (2004).

[12] Y. Sainoo et al., Surf. Sci. 536, L403 (2003); Jpn. J. Appl. Phys. 41, 4976 (2002).

[13] M. Kawai et al., Phil. Trans. R. Soc. A 362, 1163 (2004); S. Katano (personal communication).

[14] Y. Kim et al. (unpublished).

[15] Vibrational quanta for LB motion is obtained from powerlaw dependence of motion rate $(R)$ on tunneling current $(I), R \propto I^{N}$, where $N$ is the order of the motion. For LB motion, the estimated $N$ is $\sim 2$ for bias voltage $<40 \mathrm{mV}$, while $N \sim 1$ for $V s>80 \mathrm{mV}$.

[16] H. Okuyama, H. Kato, J. Yoshinobu, and M. Kawai, J. Chem. Phys. 113, 2866 (2000).

[17] B. N. J. Persson and A. Baratoff, Phys. Rev. Lett. 59, 339 (1987); R. E. Palmer and P. J. Rous, Rev. Mod. Phys. 64, 383 (1992), and references therein.

[18] M. Head-Gordon and J. C. Tully, J. Chem. Phys. 96, 3939 (1992).

[19] N. Lorente, M. Persson, L. J. Lauhon, and W. Ho, Phys. Rev. Lett. 86, 2593 (2001).

[20] H. Ueba and B. N. J. Persson, Surf. Sci. 566-568, 1 (2004). 